\title{
Spondyloepimetaphyseal dysplasia with joint laxity (Beighton type): A unique South African disorder
}

\author{
E M Honey, MB ChB, MMed (Paed) \\ Department of Genetics, Faculty of Agriculture and Natural Science, University of Pretoria, South Africa
}

Corresponding author: E M Honey (engela.honey@up.ac.za)

Spondyloepimetaphyseal dysplasia with joint laxity (SEMD-JL) is an autosomal recessive skeletal dysplasia in which stunted stature, articular hypermobility and spinal malalignment are the major manifestations. Structural cardiac abnormalities are sometimes present. Approximately 30 affected children have been recognised previously in the Afrikaans-speaking community in South Africa, and in several, mutations in the B3GALT6 gene have been incriminated. In this article, case details of three additional affected children in two families are documented, and four additional families are mentioned. The Pierre-Robin sequence and unilateral renal agenesis are previously unreported concomitants. The mutational status where known is recorded

S Afr Med J 2016;106(6 Suppl 1):S54-S56. DOI:10.7196/SAMJ.2016.v106i6.10994

Prof. Beighton has inspired me since the first day when I asked him for an opinion regarding a child with a skeletal dysplasia, back in 1999. This led to my first article in the field of medical genetics with Prof. Beighton as co-author. I have subsequently contacted him on numerous occasions for advice on patients with difficult clinical diagnoses, and he has always been so enthusiastic and helpful. I collected the group of patients with SEMD-JL and he offered genetic testing. I admire his passion and dedication to the profession and always use him as an example of a person that positively influenced my life. I therefore dedicate this article to him.

Thank you Prof. Beighton for the role you play in my career and life.

\section{Background}

Spondyloepimetaphyseal dysplasia with joint laxity 1 (Beighton type) (SEMD-JL, MIM271647) is an autosomal recessive genetic disorder present in Afrikaans-speaking South Africans of Dutch and German descent. The condition was first described in 1980 by Beighton and Kozlowski. ${ }^{[1]}$ The clinical manifestations are stunted growth associated with progressive scoliosis and multiple dislocations. Facial dysmorphism consists of a round face with a flattened nasal bridge, a button nose and long upper lip (philtrum). Additional variable features are mild skin extensibility, spatulate terminal phalanges, cleft palate, cardiac abnormalities and club feet.

Over the years, $\sim 30$ South African children with SEMD-JL have been documented. The burden of ill health is significant and few, if any, have survived into adulthood. The molecular basis of the condition, the B3GALT6 gene, was elucidated by researchers in Japan, ${ }^{[2]}$ thereby facilitating diagnostic confirmation, antenatal diagnosis and other aspects of genetic management. Subsequently, three different mutations in the B3GALT6 gene were identified in eight affected families in South Africa (SA). ${ }^{[3]}$

In the past decade, the author has had clinical contact with six children with SEMD-JL in five families domiciled in Gauteng. With the recent availability of molecular testing in SA, the mutational status of two of these families has been ascertained. The clinical features and radiological manifestations in their affected children have been described, depicted and discussed in order to alert colleagues to the presence and implications of SEMD-JL in SA.

SEMD-JL was initially documented in seven affected children following a review of accumulated clinical and radiographic data during an academic visit to the University of Cape Town (UCT) by Prof. Kazimierz Kozlowski of Sydney, Australia. ${ }^{[1]}$ Thereafter, visits to special schools for the physically handicapped and contact with colleagues in other centres in SA resulted in the identification of an additional 12 cases, and it became evident that the disorder represented a unique medical problem in this country. ${ }^{[4,5]}$ The radiographic features of SEMD-JL were subsequently analysed and depicted in detail. ${ }^{[6]}$

In 1991, in view of the relatively high frequency of SEMD-JL in $\mathrm{SA}$, an in-depth study of the ancestral lineage was undertaken using state and church archives, covering 14 generations in 8 of the affected families. Two ancestral matriarchs who lived in Western Europe during the mid-17th century were identified, thereby facilitating the recognition of obligate heterozygotes for the determinant gene in several present-day SA families. ${ }^{[7]}$ It is entirely possible that the gene reached SA from Europe on several occasions.$^{[8]}$ Further cases were recognised $^{[9]}$ and by 2010 the eponymous content of the title of the condition had been listed in the International Nosology of Genetic Skeletal Disorders 2010. ${ }^{[10]}$ This format was preserved in the current 2015 revision of the Nosology. ${ }^{[11]}$

In 2013, researchers in Japan identified mutations in the B3GALT6 gene in SEMD-JL, ${ }^{[2]}$ and as the phenotype in several of their patients was very similar to that of the affected SA children, investigation of this gene were undertaken at UCT. ${ }^{[3]}$ This molecular sequencing revealed three different mutations in the SA families, one of which was also present in the Japanese series.

\section{Case reports}

Family 1

The first daughter born to non-consanguineous Afrikaner parents was referred at the age of 5 months to the Orthopaedic Department, University of Pretoria for management of her club feet. She was born after an uncomplicated pregnancy and there was no family history apart from club feet in her paternal grandmother. On examination, she had a round face with flattened nasal bridge, large anterior fontanel, slightly blue sclerae, a long upper lip, micrognathia and a cleft palate (Fig. 1). There were contractures of the interphalangeal joints, dislocated elbows and hips, and bilateral talipes equinovarus (Fig. 2). She also had scoliosis and the diagnosis of SEMD-JL was entertained. She had an audible systolic cardiac murmur and an echocardiogram showed a $7.2 \mathrm{~mm}$ atrial septal defect (ASD) with left-to-right shunt. Sonography of the kidney revealed 
mild to moderate dilatation of the left renal pelvis and a left ureterocele. Her right hip was dislocated and the left had grade 4 dysplasia with $<50 \%$ cover of the femoral head by the acetabulum. Radiographs of the spine confirmed the presence of scoliosis and platyspondyly. The metaphyses of the long bones were widened and there were bilateral dislocations of the radial heads (Fig. 3). The cardiac shadow was enlarged. The child underwent surgical closure of the cleft palate and the ASD was treated

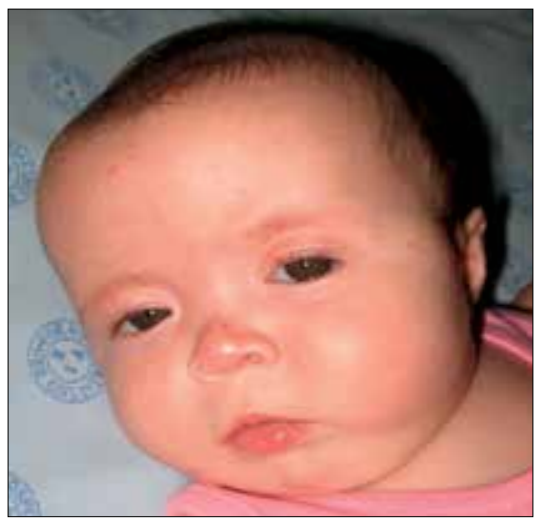

Fig. 1. Family 1. Girl aged 5 months. The characteristic button nose, long upper lip and micrognathia are evident.

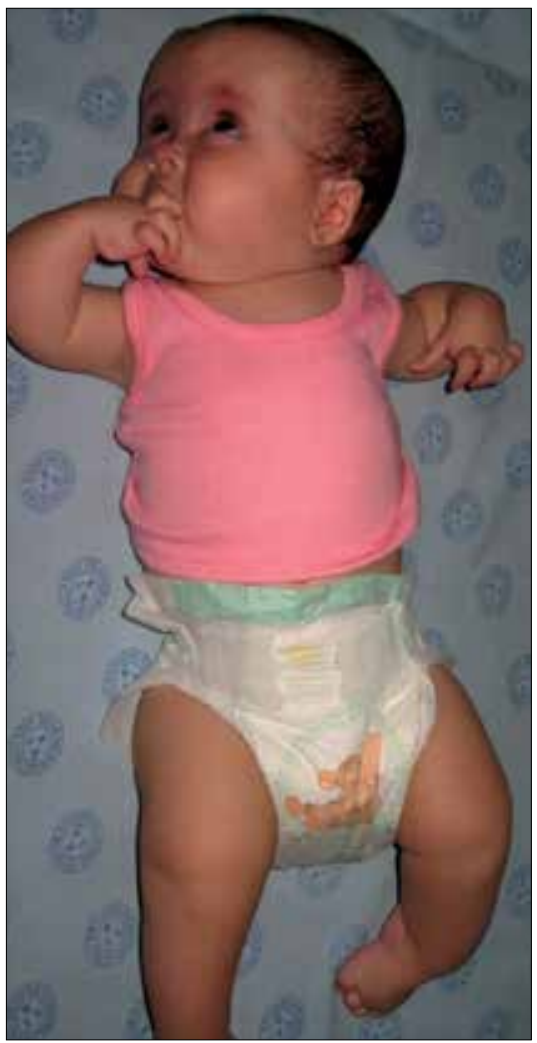

Fig. 2. Family 1. Girl aged 5 months. The elbows lack full extension due to dislocation of the radial heads. The hips are also dislocated and talipes equinovarus is present. conservatively. She had corrective surgery to her feet. The scoliosis became progressively worse and was treated with bracing. She showed marked neurodevelopmental delay and started walking only after the age of 3 years.

In a further pregnancy, antenatal ultrasound in late gestation confirmed the diagnosis of SEMD-JL. The affected male infant developed severe respiratory distress soon after birth. He had the manifestations of SEMD-JL together with severe micrognathia, glossoptosis and a cleft palate (Pierre Robin sequence). He also had contractures of his fingers and multiple dislocations. His airway was compromised and an attempt was made to perform a glossopexy, but the surgery was cancelled owing to difficulty with intubation prior to the procedure. He was nursed on his stomach but unfortunately died in the hospital at 1 month of age. Both children tested positive for compound heterozygous mutations, c. $[200 C>T] ;[235 A>G]$ in B3GALT6 and it was confirmed that the father carried the $c .200 C>T$ mutation. The mother's blood was unfortunately not available for testing. The affected sister subsequently died at the age of 4 years due to a respiratory infection.

\section{Family 2}

The second-born child from non-consanguineous parents presented at birth with clinical features consistent with the diagnosis of SEMD-JL. An ultrasound at 24 weeks gestation had shown shortening of the limbs, but the diagnosis was only made after birth. There was no relevant family history. She had

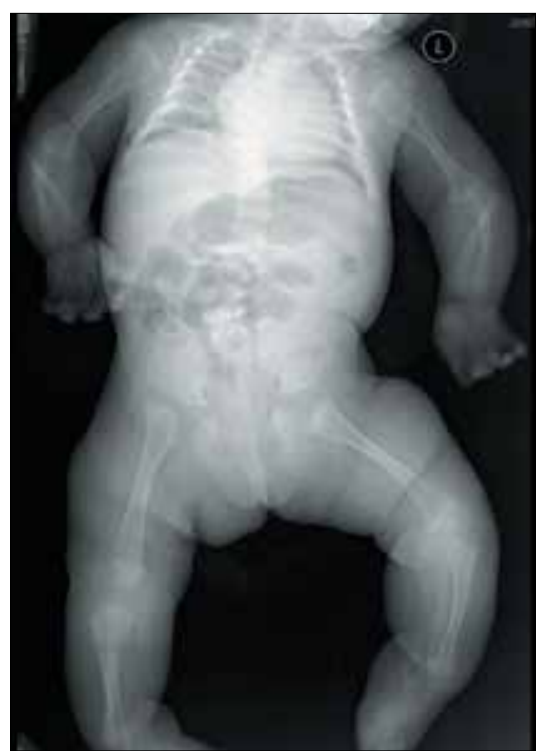

Fig. 3. Family 1. Girl aged 5 months. The key diagnostic feature of bilateral dislocation of the radial heads is evident. The metaphyses of the long bones are widened. a cleft palate as well as facial dysmorphism consisting of a flat nasal bridge, a small nose and micrognathia. There was camptodactyly of the fingers and both radial heads were dislocated. She had bilateral clubbed feet and scoliosis, unilateral renal agenesis and a normal heart apart from an open foramen ovale. The palatal cleft was repaired but she developed recurrent respiratory infections for which she needed intubation and ventilation for more than 2 weeks. Her lung capacity deteriorated to such an extent that she needed ambulatory oxygen. Her scoliosis became progressively worse and due to documented sleep apnoea, she currently needs continuous positive airway pressure while sleeping (Figs 4 - 6). She started walking with an aid at the age of 4 years and 4 months. Molecular investigations revealed compound heterozygous mutations c. $[200 C>T] ;[235 A>G]$ in B3GALT6. Her father is heterozygous for the $c .235 A>G$ mutation and her mother is heterozygous for the $c .200 C>T$ mutation.

\section{Additional families with SEMD-JL}

The author had contact with four additional SEMD-JL families prior to the availability of molecular testing

A newborn baby girl was referred for an opinion as she presented with a cleft palate, scoliosis and multiple dislocations of her joints. She had a cardiac murmur and an ASD was diagnosed. She developed cardiac failure at about 1 month of age and died shortly thereafter. Genetic diagnosis was not available at that stage.

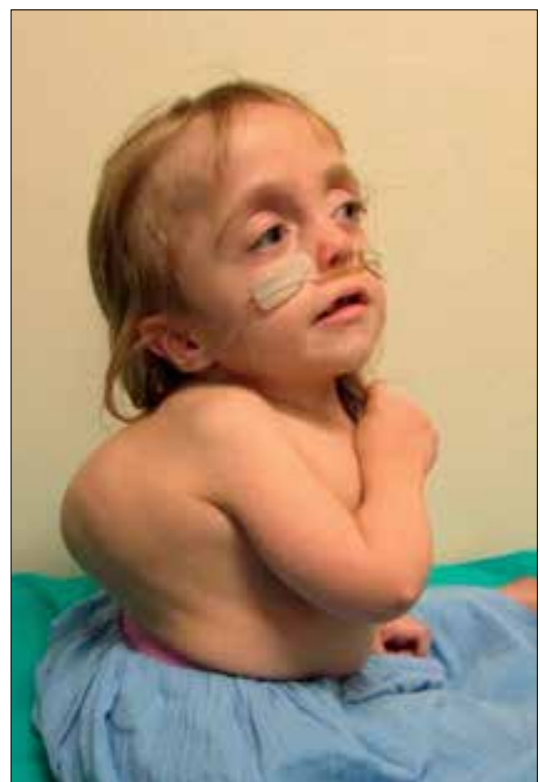

Fig. 4. Family 2. Girl aged 5 years. The typical facial features are evident. Severe progressive kyphoscoliosis has led to respiratory distress. 


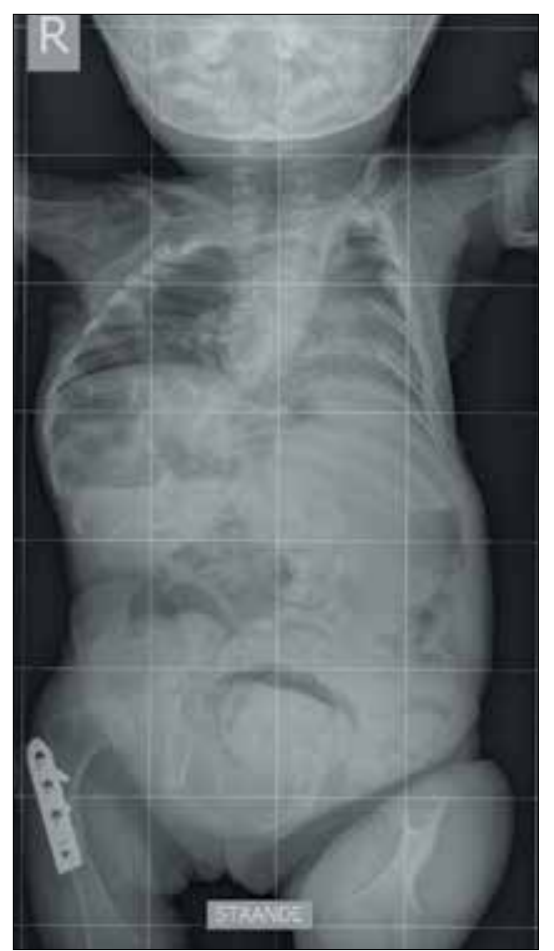

Fig. 5. Family 2. Girl aged 5 years. Anteriorposterior view. Gross platyspondyly and kyphoscoliosis are evident. The femora are very short with expanded metaphyses and delayed epiphyseal development.

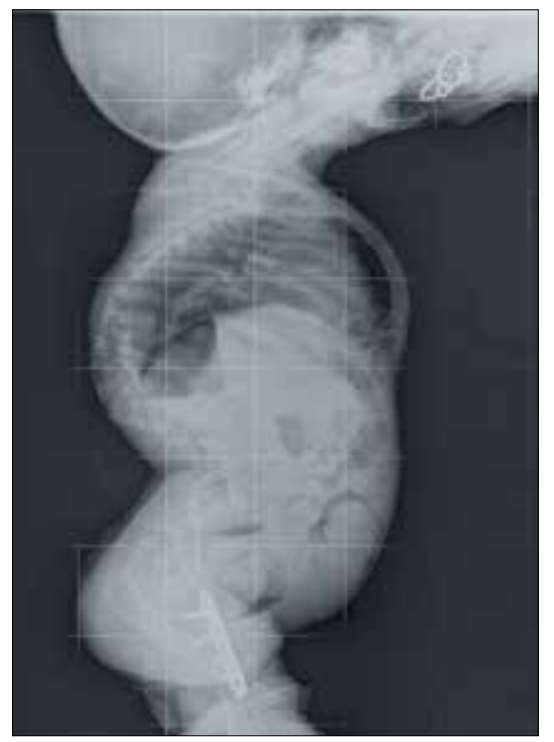

Fig. 6. Family 2. Girl aged 5 years. Lateral view. Platyspondyly and severe spinal malalignment.

Another female newborn baby was referred to the cleft lip and palate clinic for evaluation. On close inspection, she had multiple dislocations as well as a dysmorphic facies suggestive of SEMD-JL. She died at about 3 months of age due to cardiac complications.

Two other, older individuals from two separate families were lost to follow-up, but both had the clinical features of SEMD-JL and progressive scoliosis.

\section{Discussion}

In SA, SEMD-JL has only been encountered in persons with close genetic links to the Afrikaans-speaking community. The precise incidence in this group is unknown, but in addition to the 30 documented cases it is likely that others have remained undiagnosed, especially affected infants who died in the neonatal period. A reasonable estimate would be the birth of an affected child every year in this country.

In the neonate, early diagnosis facilitates prognostication and appropriate medical and genetic management (Table 1). Gross joint laxity and sequelae such as club feet, dislocated hips and spinal malalignment are important diagnostic indicators. Bilateral dislocation of the radial heads is usual and results in lack of full extension and rotation at the elbow joint. This paradoxical limitation in an otherwise hypermobile infant is an important diagnostic sign. Recognition of the characteristic facies clinches the diagnosis. Radiographic studies demonstrate the typical dislocation of the radial heads, which may be associated with shortening and curvature of the radius. Imaging at this stage is otherwise nonspecific. In later childhood, the characteristic generalised epiphyseal, metaphyseal and vertebral changes will be evident.

The primary phenotype in SEMD-JL is essentially the result of the interaction of joint laxity and skeletal dysplasia. Variable concomitants include facial and palatal clefts and structural cardiac abnormalities. The intellect seems normal in most of the cases although children commonly present with neurodevelopmental delay. Progressive kyphoscoliosis and cardiac failure due to septal defects are the disabling abnormalities that cause high morbidity and early mortality. The life span is markedly reduced and most patients die before the end of the second decade.

Previously unrecognised malformations that were documented in the case reports in this article were the Pierre Robin sequence in one infant and unilateral renal agenesis with dilatation of the renal pelvis and an ureterocele in the other.

The recognition of the causative role in mutations in the B3GALT6 gene represents a major breakthrough in the understanding of SEMD-JL. Prenatal diagnosis by ultrasound was previously the only option, but the abnormal skeletal features are usually only visible during late pregnancy. In the near
Table 1. Diagnostic manifestations of SEMD-JL in infancy

\begin{tabular}{l}
\hline Facies \\
Round face \\
Button nose \\
Long upper lip \\
Joints - generalised hypermobility \\
Dislocated radial heads \\
Dislocated hips, talipes equino varus - \\
variable \\
Scoliosis - progressive \\
Cardiac malformation - variable and \\
inconsistent \\
Skin - soft and hyperextensible \\
Radiological \\
Bilateral dislocation of the radial heads \\
Metaphyseal widening - mild \\
Platyspondyly with scoliosis - variable
\end{tabular}

future with molecular technology, early prenatal diagnosis will be available for families with a history of SEMD-JL.

Written permission was given by the parents for the case details and publication of the photos.

Acknowledgements. The author wants to thank A A Vorster and R S Ramesar (Division of Human Genetics, University of Cape Town) for the molecular studies done on the two families.

\section{References}

1. Beighton P, Kozlowski K. Spondylo-epi-metaphyseal dysplasia with joint laxity and severe, progressive kyphoscoliosis. Skeletal Radiol 1980;5(4):205-212. DOI:10.1007/bf00580591

2. Nakajima M, Mizumoto S, Miyake N, et al. Mutations in . Nakajima M, Mizumoto S, Miyake $\mathrm{N}$, et al. Mutations in
B3GALT6, which encodes a glycosaminoglycan linker region enzyme, cause a spectrum of skeletal and connective tissue disorders. Am J Hum Genet 2013;92(6):927-934. DOI:10.1016/j. ajhg.2013.04.003

3. Vorster AA, Beighton P, Ramesar RS. Spondyloepimetaphyseal dysplasia with joint laxity (Beighton type); mutation analysis in eight affected South African families. Clin Genet 2015;87(5):492495. DOI:10.1111/cge.12413

4. Beighton P, Kozlowski K, Gericke G, Wallis G, Grobler L. Spondylo-epimetaphyseal dysplasia with joint laxity and severe, progressive kyphoscoliosis. A potentially lethal dwarfing disorder. S Afr Med J 1983;64(20):772-775.

5. Beighton P, Gericke G, Kozlowski K, Grobler L. The manifestations and natural history of spondylo-epi-metaphyseal festations and natural history of spondylo-epi-metap
dysplasia with joint laxity. Clin Genet 1984;26(4):308-317.

dysplasia with joint laxity. Clin Genet 1984;26(4):308-317.
6. Kozlowski K, Beighton P. Radiographic features of spondyloKozlowski K, Beighton P. Radiographic features of spondylo-
epimetaphyseal dysplasia with joint laxity and progressive kyphoscoliosis. Review of 19 cases. Rofo 1984;141(3):337341

7. Torrington M, Beighton P. The ancestery of spondyloepimetaphyseal dysplasia with joint laxity (SEMDJL) in South Africa. Clin Genet 1991;39(3):210-213.

8. Beighton P. Spondyloepimetaphyseal dysplasia with joint laxity (SEMDJL). J Med Genet 1994;31(2):136-140.

9. Christianson AL, Beighton P. Spondyloepimetaphyseal dysplasia with joint laxity (SEMDJL) in three neonates. Genet Couns 1996;7(3):219-225.

10. Warman ML, Cormier-Daire V, Hall C, et al. Nosology and classification of genetic skeletal disorders: 2010 revision. classification of genetic skeletal disorders: 2010 revision.
Am J Med Genet A 2011;155A(5):943-968. DOI:10.1002/ Am J Med

11. Bonafe L, Cormier-Daire V, Hall C, et al. Nosology and Bonafe L, Cormier-Daire V, Hall C, et al. Nosology and
classification of genetic skeletal disorders: 2015 revision. Am J Med Genet A 2015;167(12):2869-2892. DOI:10.1002/ ajmg.a.37365 